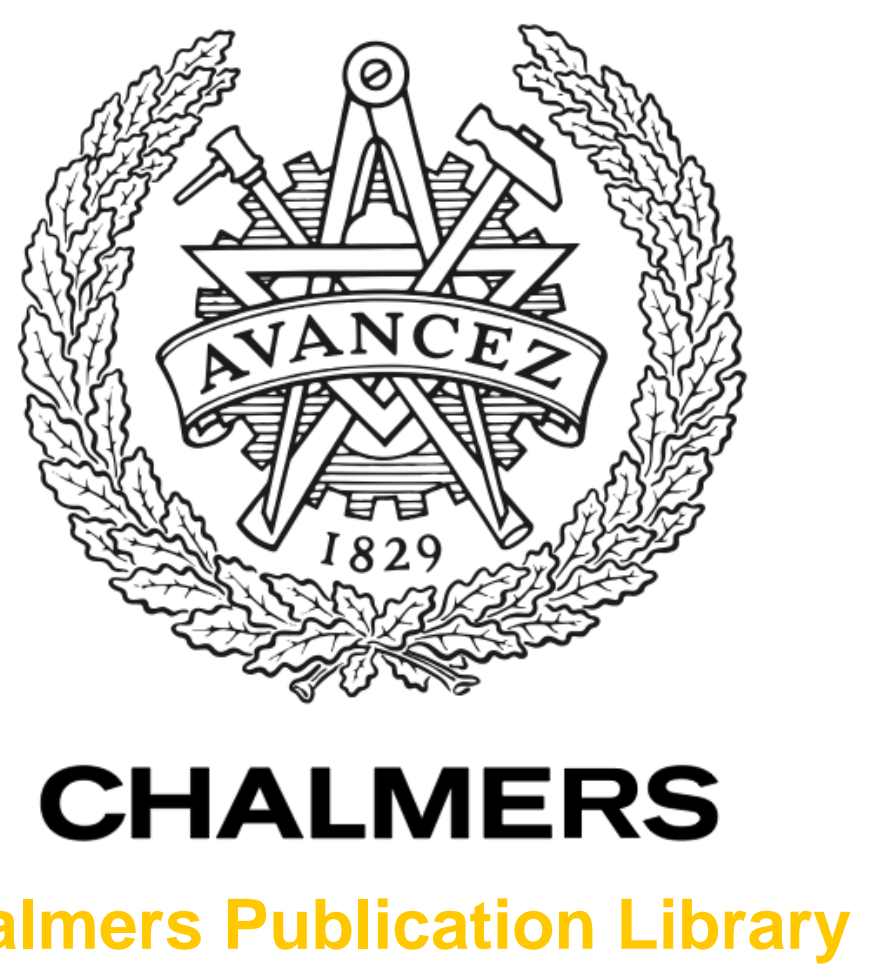

\title{
Wideband Planar Hybrid With Ultralow Amplitude Imbalance
}

This document has been downloaded from Chalmers Publication Library (CPL). It is the author's version of a work that was accepted for publication in:

IEEE Microwave and Wireless Components Letters (ISSN: 1531-1309)

Citation for the published paper:

Rashid, H. ; Yadranjee Aghdam, P. ; Meledin, D. et al. (2017) "Wideband Planar Hybrid

With Ultralow Amplitude Imbalance". IEEE Microwave and Wireless Components Letters, vol. 27(3), pp. 230-232.

Downloaded from: http://publications.lib.chalmers.se/publication/248520

Notice: Changes introduced as a result of publishing processes such as copy-editing and formatting may not be reflected in this document. For a definitive version of this work, please refer to the published source. Please note that access to the published version might require a subscription.

Chalmers Publication Library (CPL) offers the possibility of retrieving research publications produced at Chalmers University of Technology. It covers all types of publications: articles, dissertations, licentiate theses, masters theses, conference papers, reports etc. Since 2006 it is the official tool for Chalmers official publication statistics. To ensure that Chalmers research results are disseminated as widely as possible, an Open Access Policy has been adopted.

The CPL service is administrated and maintained by Chalmers Library. 


\title{
Wideband Planar Hybrid with Ultra-Low Amplitude Imbalance
}

\author{
Hawal Rashid, Parisa Yadranjee Aghdam, Denis Meledin, Vincent Desmaris, Member, IEEE, and \\ Victor Belitsky, Senior Member, IEEE
}

\begin{abstract}
We present a new design approach for the $90^{\circ}$ directional coupler with very low amplitude imbalance. The primary feature of this quadrature coupler is the introduction of a controllable ripple in the operational band for achieving a better overall amplitude balance. This design concept is demonstrated through a $90^{\circ}$ branch-line hybrid for the $4-7.9$ GHz band $(65 \%$ fractional bandwidth) using microstrip transmission lines. Our simulations indicate that the amplitude imbalance of the designed hybrid is better than $0.3 \mathrm{~dB}$ over the most of the $4-7.9 \mathrm{GHz}$ band with a phase imbalance better than $\pm 8.5^{\circ}$. Experimental verification of the hybrid shows excellent agreement with simulations.
\end{abstract}

Index Terms-Directional couplers, Branch line couplers, wideband hybrids

\section{INTRODUCTION}

$\mathrm{D}$ IRECTIONAL couplers have been studied since the 1940s, resulting in a variety of design techniques for both waveguide and substrate-based components. The $3 \mathrm{~dB}$ coupler, also commonly referred to as a hybrid is a special case of the directional coupler that provides equal power division/combination and $90^{\circ}$ or $180^{\circ}$ phase difference between the outputs depending on the design topology. The $3 \mathrm{~dB}$ coupler is one of the basic circuit components for balanced amplifiers [1], sideband separating (2SB) mixers [2], array antennas, filters, and more. There are many design topologies of the substrate-based hybrids with diverse fabrication complexity that offer improved hybrid performance [3], [4].

Nonetheless, the $90^{\circ}$ branch line hybrids $(\mathrm{BLH})$ remain a pertinent research topic even today due to their simplicity and ease of fabrication [5].

The standard BLH has a relatively narrow fractional bandwidth (FBW) of approximately $20 \%$ for an amplitude imbalance of $0.5 \mathrm{~dB}$, which is a major limitation of the coupler. Multi-section techniques have been suggested in order to increase the bandwidth of the coupler [6]. The required branch impedance rapidly increases with every additional branch, which limits the multi-section branch line coupler to 4-5 branches, and therefore the fractional bandwidth of the BHL [6], depending on the dielectric constant, the thickness of the substrate and the used fabrication technique. There are a variety of designs apart from the multi-section technique that offer increased bandwidth characteristics of the BLH [7], [8], [9]. However, the performance of the wideband branch-line hybrids varies

Manuscript submitted on February 8, 2016. The authors are with the Group of Advanced Receiver Development (GARD) at Chalmers University of Technology,Gothenburg,Sweden.(email:Hawal.rashid@gmail.com) substantially from one design to another. Typically, these designs have adequate isolation and return loss whereas the amplitude imbalance is often larger than 1-2 dB. Furthermore, the FBW of these designs would be substantially reduced when compared with the wideband designs with small amplitude imbalance.

A typical design condition of the branch line hybrid is a maximum isolation [10]. Similar to [11], the work presented here allows very small amplitude imbalance at the cost of some degradation of the isolation, return loss and phase difference. This is achieved by loosening the condition for maximum isolation and modifying the hybrid such that interleaved ripples within the operating frequency band are introduced into the through and coupled signals, thus yielding minimum amplitude imbalance. Such a component would is likely to be used in balanced topologies e.g. sideband separating mixers used in radio astronomy, where the symmetry of the component is more important than absolute matching at each port.

The hybrid design allows performance similar to the more complex designs [1], [6], [7] i.e., substantially larger bandwidth than the corresponding single section design. However, the design presented here is planar using microstrip transmission lines, which makes it easier to integrate with other components. Furthermore, the design is also more reliable and cheaper to produce than the more complex designs.

\section{HYBRID DESIGN}

\section{A. Design of the modified branch-line hybrid}

In order to generate ripples in the operation band, the condition for perfect isolation needs to be relaxed. This means that small reflections inside the hybrid need to be deliberately created. However, imposing the condition that the reciprocity of the four port network must be preserved suggests that the mismatch need to be symmetrically located within the hybrid layout of any odd number of sections.

There are different arrangements of the BLH that could satisfy the abovementioned conditions, for example a four branch BLH layout (see Fig.1) similar to [6] could be used. However, for the intended design goals, the design values provided in [6] cannot be used since they provide amplitude imbalance of $0.86 \mathrm{~dB}$ with no ripple within the operating frequency band.

Unlike [6], the proposed design intends to minimize the amplitude imbalance over a large FBW by sacrificing isolation in exchange for interleaved ripples of the coupled and through signals. This design approach not only reduces the amplitude imbalance substantially, but it also substantially lowers the amplitude imbalance at the center frequency. The performance enhancement comes at the cost of slightly degraded phase imbalance. Similarly, the 
impedance mismatch between the center section and the adjacent sections deteriorates the return loss and isolation as shown [12].

For the design layout presented in Fig 1, generating ripples in the passband obviously requires that the impedance, $Z_{4}$ should be lower than $Z_{3}$. Since the multisection hybrid consists of several subnetworks with different couplings [3], the three section BLH can thought of approximately as two $8.34 \mathrm{~dB}\left(Z_{1}=Z_{2}\right.$ for each 8.34 coupler) cascaded with a quarter-wavelength interconnecting transmission line with characteristic impedance $Z_{4}\left(Z_{4}=Z_{3}\right.$ in the case of two $8.34 \mathrm{~dB}$ couplers). Furthermore, the characteristic impedance of a coupler with perfect isolation could be found by employing the even and odd mode analysis technique [10], where the ABCD matrix parameters $\mathrm{B}$ are equalized i.e., $B=C$. In this case, the characteristic impedance $Z_{l}\left(Z_{2}=Z_{l}\right.$ in Fig. 1$)$ and $Z_{3}$ are given by:

$$
\begin{aligned}
& Z_{3}=\sqrt{1-10^{-C / 10}} \\
& Z_{1}=\sqrt{\frac{Z_{3}^{2}}{1-Z_{3}^{2}}}
\end{aligned}
$$

where $\mathrm{C}$ is the coupling in $\mathrm{dB}$.

The design of the proposed hybrid can be carried out through, e.g., an iterative process. The first step is to calculate the characteristic impedance of the $8.34 \mathrm{~dB}$ coupler sections, and the second step is to tune the characteristic impedance of the interconnecting transmission lines in order to generate small reflections and thus controllable ripples in the passband. The overall coupling is then fine-tuned by slightly adjusting the impedance of the coupler itself i.e., adjusting the characteristic impedances $Z_{1}$ and $Z_{3}$ until the desired amplitude and phase imbalance characteristics vs. trade-offs are reached (isolation and return loss). Furthermore, the greater the characteristic impedance mismatch between $\mathrm{Z}_{4}$ and $\mathrm{Z}_{3}$ becomes $\left(\mathrm{Z}_{4}<\mathrm{Z}_{3}\right)$, the larger the ripple amplitude becomes and thus increasing the bandwidth of the hybrid. However, the gain in bandwidth comes at the cost of further impedance mismatch and degradation of the phase imbalance [12].

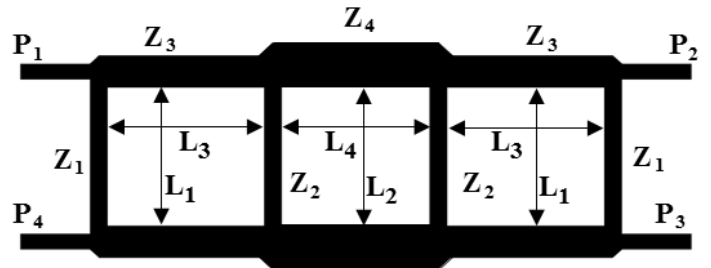

Fig. 1: A three section branch-line coupler with equal branch impedance values.

\section{B. Implementation for the 4-7.9 $\mathrm{GHz}$ operation band}

The iterative process was carried out in the Keysight ADS [13] circuit simulator. The design was later simulated in the ADS Momentum as a final check. The design values of the proposed hybrid are obtained for a 20 mil thick alumina substrate covering a FBW of $65 \%$ i.e., $4-7.9 \mathrm{GHz}$ with the maximum amplitude imbalance, phase difference, return loss/isolation of $0.3 \mathrm{~dB}, 90 \pm 8.5^{\circ}$, and $12 \mathrm{~dB}$ respectively.

According to the Keysight ADS circuit simulator, the directivity for this specific FBW is approximately $9 \mathrm{~dB}$. However, simulations show that the directivity for a FBW of $55 \%$ is better than $10 \mathrm{~dB}$. In this case, the phase imbalance improves as well. The return loss and isolation of this design approach is comparable (if not slightly higher) to [7] for a FBW of $40 \%$, whereas the amplitude imbalance and phase imbalance is better than $0.1 \mathrm{~dB}$ and $\pm 4^{\circ}$ respectively, the design values are summarized in table I. The design values of the demonstrator $(65 \%$ FBW $)$ branch-line hybrid impedances, microstrip line widths and lengths are tabulated in table II, whereas some of the performance characteristics are tabulated in table III.

The hybrid was fabricated using one lithography step, the microstrip lines and the back side of the alumina chip were gold-plated with approximately $4 \mu \mathrm{m}$ thick gold.

\section{TABLE I}

THE BLH FOR 42\% FBW USING THE PRESENTED APPROACH.

\begin{tabular}{llr}
\hline \hline $\mathrm{N}$ & $Z_{N}(\Omega)$ & $L_{N}(m)$ \\
\hline 0 & 50 & \\
1 & 127.6 & $\lambda / 4$ \\
2 & 127.6 & $\lambda / 4$ \\
3 & 49.2 & $\lambda / 4$ \\
4 & 43.2 & $\lambda / 4$
\end{tabular}

\section{MEASUREMENTS}

The hybrid was measured with a four port Vector Network Analyzer (VNA). The VNA was calibrated with a 4 port UOSM (Unknown, Open-circuit, Short-circuit and Matched) standards. Fig. 3 and 4 show the simulated and measured performance of the proposed hybrid. It can be seen from Fig.3 that the coupling of the measured hybrid is slightly lower as compared to the simulated coupling. Consequently, the amplitude imbalance of the simulated hybrid is $0.1 \mathrm{~dB}$ $(0.2 \mathrm{~dB}$ at the center frequency) larger as compared to the measured amplitude imbalance and the bandwidth of the fabricated hybrid is somewhat narrower.

TABLE II

THE DIMENSIONS OF THE MEASURED BRANCH LINE HYBRID.

\begin{tabular}{llll}
\hline \hline $\mathrm{N}$ & $Z_{N}(\Omega)$ & $W_{N}(\mu \mathrm{m})$ & $L_{N}(\mu \mathrm{m})$ \\
\hline 0 & 50 & 490 & 1000 \\
1 & 109.6 & 45.5 & 5438 \\
2 & 109.6 & 45.5 & 5051 \\
3 & 40.6 & 743 & 4759 \\
4 & 30.6 & 1130 & 4840
\end{tabular}

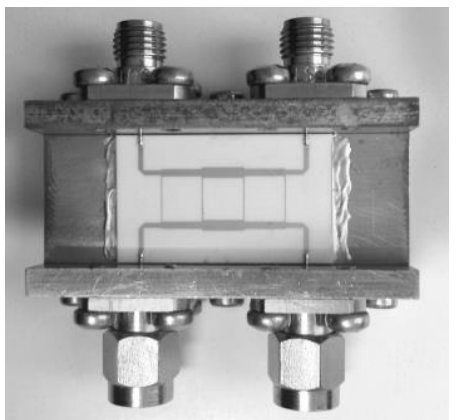

Fig. 2. Photograph of the measured branch-line hybrid. The hybrid Alumina substrate was glued to the fixture by a conducting epoxy.

We would like to note that this very small difference could be as well due to the transitions from microstrip-tocoaxial line and the SMA connectors themselves, which were not included in the simulations. Still, the measured performance shows excellent agreement with the simulated performance and demonstrates amplitude imbalance better than $0.3 \mathrm{~dB}$ over most of the band and phase difference 
better than $90^{\circ} \pm 8.5^{\circ}$. The isolation and return loss are better than $12 \mathrm{~dB}$. The measured factional bandwidth is approximately $65 \%$.

The performance characteristics of the hybrid presented here is compared with other wideband branch-line hybrids in Table III. The novel planar design approach presented here shows very large bandwidth and at the same time very small amplitude imbalance with adequate isolation and return loss values. The performance characteristics of the presented hybrid are superior to the designs presented in Table III given the bandwidth and amplitude imbalance.

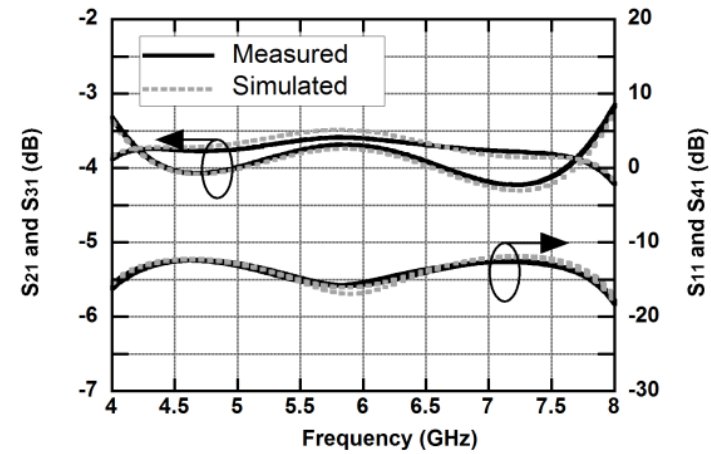

Fig. 3. Measured through, coupled, reflection and isolation performance of the proposed design.

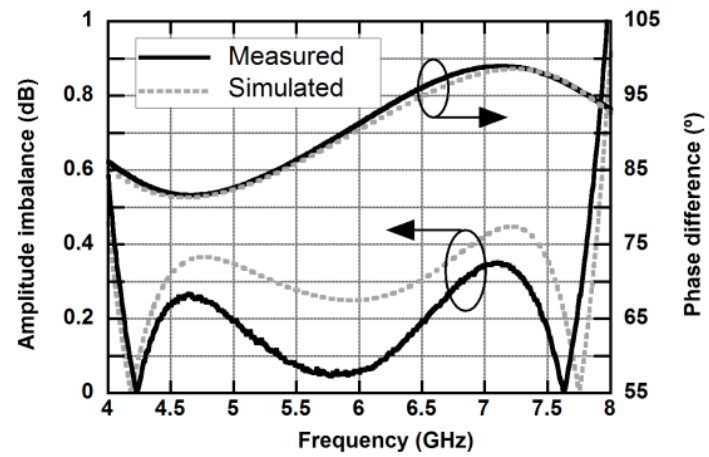

Fig. 4. Measured and simulated amplitude and phase imbalance of the proposed design.

\section{TABLE III}

HYBRID PERFORMANCE FOR DIFFERENT REFERENCES ( $\triangle \mathrm{A}$ IS THE AMPLITUDE IMBALANCE, $\Delta \varphi$ IS THE PHASE DIFFERENCE, AND RL IS THE RETURN LOSS, THE SIZE REFERS ONLY TO THE HYBRID AND NOT THE SUBSTRATE SIZE)

\begin{tabular}{|c|c|c|c|c|c|c|}
\hline paper & $\begin{array}{l}\text { BW } \\
(\%)\end{array}$ & $\begin{array}{c}\mathrm{RL} \\
(\mathrm{dB})\end{array}$ & $\begin{array}{l}\text { Isolation } \\
\text { (dB) }\end{array}$ & $\begin{array}{c}\Delta \mathrm{A} \\
(\mathrm{dB})\end{array}$ & $\Delta \varphi$ & Size \\
\hline [5] & 76 & $>10$ & $>10$ & \pm 1.8 & $\begin{array}{r}90 \pm \\
15^{\circ}\end{array}$ & $\begin{array}{c}0.43 \cdot 2 \lambda \times \\
\lambda / 2\end{array}$ \\
\hline [7] & 40 & $>15$ & $>17$ & \pm 0.1 & NA & $5 \lambda / 4 \times \lambda / 2$ \\
\hline [8] & 49 & $>15$ & $>15$ & \pm 3 & NA & $3 \lambda / 8 \times \lambda / 4$ \\
\hline [9] & 30 & $>20$ & $>20$ & \pm 0.5 & NA & $3 \lambda / 4 \times \lambda / 4$ \\
\hline [14] & 75 & $>17$ & $>17$ & \pm 1 & \pm 2 & $\begin{array}{c}0.2 \cdot 3 \lambda / 4 \times \\
\lambda / 4\end{array}$ \\
\hline $\begin{array}{l}\text { This } \\
\text { work }\end{array}$ & 65 & $>12$ & $>12$ & \pm 0.3 & $\begin{array}{l}90 \pm \\
8.5^{\circ}\end{array}$ & $3 \lambda / 4 \times \lambda / 4$ \\
\hline $\begin{array}{c}\text { Simula } \\
\text { ted }\end{array}$ & 42 & $>15$ & $>15$ & \pm 0.1 & $\begin{array}{l}90 \pm \\
3.5^{\circ}\end{array}$ & $3 \lambda / 4 \times \lambda / 4$ \\
\hline
\end{tabular}

From table III it can be seen that the performance that could be obtained with the design approach presented here is similar or better than the other designs in table III. Specifically, the presented design is much better than [5] in terms of amplitude and phase imbalance and size, but also in terms of RL, isolation and directivity. Furthermore, the suggested design for $40 \%$ fractional bandwidth is similar to [7] in performance, but a half wavelength smaller (cf. Table III).

\section{CONCLUSIONS}

We present a new design approach for the $90^{\circ}$ planar hybrid and its implementation. The primary feature of this quadrature hybrid is the introduction of a controllable ripple in the operational band for achieving a better overall amplitude imbalance. This design concept is verified by implementation of a $90^{\circ}$ hybrid for the $4-7.9 \mathrm{GHz}$ band using microstrip transmission lines. Our simulations indicate that the amplitude imbalance of the designed hybrid is better than $0.3 \mathrm{~dB}$ over the most of the $4-7.9 \mathrm{GHz}$ band with a phase imbalance better than $\pm 8.5^{\circ}$. Experimental verification of the hybrid shows excellent agreement with simulations.

\section{ACKNOWLEDGMENT}

The authors would like to thank Sven-Erik Ferm and Mathias Fredrixon for the assistance in fabrication of the hybrid assembly.

\section{References}

I. Malo-Gomez, J. D. Gallego-Puyol, C. Diez-Gonzalez, I. Lopez-Fernandez, and C. Briso-Rodriguez, "Cryogenic Hybrid Coupler for Ultra-Low-Noise Radio Astronomy Balanced Amplifiers," Microwave Theory and Techniques, IEEE Transactions on, vol. 57, pp. 3239-3245, 2009.

J. W. Kooi, R. A. Chamberlin, R. Monje, B. Force, D. Miller, and T. G. Phillips, "Balanced Receiver Technology Development for the Caltech Submillimeter Observatory," Terahertz Science and Technology, IEEE Transactions on, vol. 2, pp. 71-82, 2012.

E. G. Cristal and L. Young, "Theory and Tables of Optimum Symmetrical TEM-Mode Coupled-Transmission-Line Directional Couplers," Microwave Theory and Techniques, IEEE Transactions on, vol. 13, pp. 544-558, 1965.

H. Rashid, D. Meledin, V. Desmaris, A. Pavolotsky, and V. Belitsky, "Superconducting 4-8 GHz Hybrid Assembly for 2SB Cryogenic THz Receivers," Terahertz Science and Technology, IEEE Transactions on, vol. 4, pp. 193-200, 2014.

N. A. Sutton, J. M. Oliver, and D. S. Filipovic, "Wideband 18$40 \mathrm{GHz}$ Surface Micromachined Branchline Quadrature Hybrid," Microwave and Wireless Components Letters, IEEE, vol. 22, pp. 462-464, 2012.

M. Muraguchi, T. Yukitake, and Y. Naito, "Optimum Design of 3-Db Branch-Line Couplers Using Microstrip Lines," Microwave Theory and Techniques, IEEE Transactions on, vol. 31, pp. 674-678, 1983.

B. Mayer and R. Knochel, "Branchline-couplers with improved design flexibility and broad bandwidth," in Microwave Symposium Digest, 1990., IEEE MTT-S International, 1990, pp. 391-394 vol.1.

B. M. Alqahtani, A. F. Sheta, and M. A. Alkanhal, "New compact wide-band branch-line couplers," in Microwave Conference, 2009. EuMC 2009. European, 2009, pp. 11591162 .

T. Kawai, H. Taniguchi, I. Ohta, and A. Enokihara, "Broadband branch-line coupler with arbitrary power split ratio utilizing microstrip series stubs," in Microwave Conference (EuMC), 2010 European, 2010, pp. 1170-1173.

J. Reed and G. J. Wheeler, "A Method of Analysis of Symmetrical Four-Port Networks," Microwave Theory and Techniques, IRE Transactions on, vol. 4, pp. 246-252, 1956. H. Rashid, D. Meledin, V. Desmaris, and V. Belitsky, "Novel Waveguide 3 dB Hybrid With Improved Amplitude Imbalance," Microwave and Wireless Components Letters, IEEE, vol. 24, pp. 212-214, 2014

[12] H. Rashid, V. Desmaris, V. Belitsky, M. Ruf, T. Bednorz, and A. Henkel, "Design of Wideband Waveguide Hybrid With Ultra-Low Amplitude Imbalance," IEEE Transactions on Terahertz Science and Technology, vol. 6, pp. 83-90, 2016.

[13] www.keysight.com

[14] R. K. Barik, K. V. P. Kumar, and S. S. Karthikeyan, "Compact wideband $3 \mathrm{~dB}$ branch line coupler with multiple symmetric PI section," in Microwave Conference (EuMC), 2015 European, 2015, pp. 275-278. 